Running title: Testosterone increases facial femininity preference

\title{
Single dose testosterone administration increases men's facial femininity preference in a Chinese population
}
Chengyang Han ${ }^{\text {a, b }}$, Yinhua Zhang a, b, Xue Lei ${ }^{\mathrm{c}}$, Xiangqian Li ${ }^{\mathrm{d}}$, Edward R. Morrison ${ }^{\mathrm{e}}$, Yin $\mathrm{Wu}^{\mathrm{a}, \mathrm{b}}$ *

${ }^{a}$ Shenzhen Key Laboratory of Affective and Social Cognitive Science, Shenzhen University, Shenzhen, China

${ }^{\mathrm{b}}$ School of Psychology, Shenzhen University, Shenzhen, China

${ }^{c}$ School of Psychology \& Neuroscience, University of St Andrews, St Andrews, United Kingdom

${ }^{\mathrm{d}}$ Department of Psychology, School of Social Development and Public Policy, Fudan University, Shanghai, China

${ }^{\mathrm{e}}$ Centre for Comparative and Evolutionary Psychology, University of Portsmouth, Portsmouth, UK

Word count: 2211, figure count: 1 , table count: 1 , references count: 21

${ }^{*}$ Correspondence: Dr. Yin Wu, School of Psychology, Shenzhen University, Shenzhen, China. Tel: +86-755-26532350 E-mail: yinwu0407@gmail.com 


\title{
Highlights
}

- Testosterone administration increases men's facial femininity preferences.

- Chinese men preferred femininity over masculinity in women's faces.

- Testosterone has a causal effect on men's facial preferences.

\begin{abstract}
Sex hormones are thought to influence human mate preferences. Previous studies have reported mixed results regarding the association between men's testosterone levels and their mate preferences. The present study investigated the effect of testosterone administration on men's facial femininity preference. Heterosexual Chinese male participants $(n=140)$ received a single dose of $150 \mathrm{mg}$ testosterone or placebo gel in a double-blind, placebo-controlled, between-participant design. Results showed that Chinese men demonstrated general preferences for feminized women's faces, consistent with previous results from the Western population. More importantly, men showed stronger attraction to femininity in women's faces three hours after testosterone administration than at the beginning of the session. In the placebo group, no significant change in facial femininity preferences was found between time points. These results indicate that exogenous testosterone increases men's facial femininity preferences in a Chinese population.
\end{abstract}

Keywords: testosterone; facial preferences; sexual dimorphism; mate preferences; attractiveness

\section{Introduction}

Many studies have found that exaggerated sex typicality in human faces (masculinity/femininity) is positively associated with facial attractiveness (reviewed in Little, Jones, \& DeBruine, 2011). This association is thought to reflect the evolutionary history of mate selection. Evidence indicates that women's sexually dimorphic facial traits signal health and fertility advantages that are preferred traits in mate selection (reviewed in Scott et al., 2014; Little et al., 2011). 
Recent research has demonstrated that the preference for sexually dimorphic features may shift as sex hormone levels fluctuate. For example, facial masculinity preferences track changes in hormone levels over the menstrual cycle in women (see Gildersleeve, Haselton, \& Fales, 2014 for a review, but see Jones et al., 2018; Marcinkowska, Galbarczyk, \& Jasienska, 2018; Marcinkowska, Kaminski, Little, \& Jasienska, 2018; Wood, Kressel, Joshi, \& Louie, 2014). The stronger attraction to masculine men at the peak fertile phase in women is thought to be an adaptation for increasing offspring health, as men's masculine features may signal heritable health (see Gangestad \& Thornhill, 2008 for a review).

Men's facial femininity preferences could be influenced by testosterone levels. Early work found that men's facial femininity preferences are positively associated with their testosterone levels (Welling et al., 2008). Welling and colleagues (2013) reported that male winners of a video game showed greater preferences for facial femininity than losers, testosterone levels being thought to increase in winners relative to losers. These results suggest that increased testosterone levels in men may lead to greater preferences for facial femininity.

Mating context can also affect men's facial femininity preference and possibly interact with the effect of testosterone. Men tend to have a stronger preference for facial femininity in the short-term rather than long-term mating context (Welling et al., 2013). A short-term mating context refers to a romantic relationship that may not last for long time, such as one-night stand; whereas a long-term mating context refers to a romantic relationship that may last for long time, such as marriage. A recent study reported that experimentally increasing testosterone levels caused men's facial femininity preference to decrease in the long-term mating context but stay constant in short-term mating context (Bird et al., 2016). This negative association between testosterone levels and men's facial femininity preferences contradicted previous findings showing a positive association between testosterone levels and men's facial femininity preference (Welling et al., 2008), although Welling et al. (2008) did not include mating context in their design. 
Regarding the mixed results in the literature, we aimed to further investigate the effect of exogenous testosterone on men's preference for femininity on women's faces in different mating contexts. The present study used a double-blind, placebo-controlled, between-participant design. Furthermore, recent research has shown cultural differences in preferences for sexually dimorphic faces (DeBruine, Jones, Crawford, Welling, \& Little, 2010; Marcinkowska et al., 2014; Scott et al., 2014), suggesting the previous results based on the Western population might not simply generalize to other populations. Therefore, the current study tested Chinese male participants and aimed to provide evidence from a non-Western population as to whether hormone levels affect facial preference in the Chinese population.

\section{Methods}

\subsection{Participants}

We recruited 140 healthy Chinese male student volunteers (mean age $=20.48$ years, $\mathrm{SD}=$ 1.68; age range $=18-25$ ) from Shenzhen University to participate in this study. Individuals who are having any psychiatric/neurological disorders or taking psychotropic medications were not eligible to participate. All participants reported they were heterosexual. Participants were asked to abstain from smoking, caffeine intake, and alcohol for $24 \mathrm{~h}$ before the experimental session. This study was conducted following the Declaration of Helsinki and was approved by the Medical Research Ethics Committee at Shenzhen University. All participants provided written informed consent. Participants were paid 45 Chinese yuan per hour. Data from four participants were lost due to computer malfunction. Thus, our final sample size for analyses was 136 .

\subsection{Stimuli}

In line with previous studies investigating sexually-dimorphic face preference (e.g., Bird et al., 2016; Welling et al., 2008), we used prototype-based image transformations to objectively manipulate sexual dimorphism of 2D shape only in face images. Skin color and texture were kept the same as the original face image. Specifically, we manufactured female and male prototype (i.e., average) face by using established computer graphic methods that have been widely used in studies of face perception (e.g., Welling et al., 2008; Bird et al., 2016). Fifty 
Chinese female faces and fifty Chinese male faces were used to make one female and one male prototype face, respectively. Next, $50 \%$ of the linear differences in $2 \mathrm{D}$ shape between symmetrized female and male prototypes were either added or subtracted from fifty young Chinese female face images. By following this protocol, we created fifty pairs of female face images (See Fig. 1A for an example of masculinized and feminized stimuli).

(A)



(B)

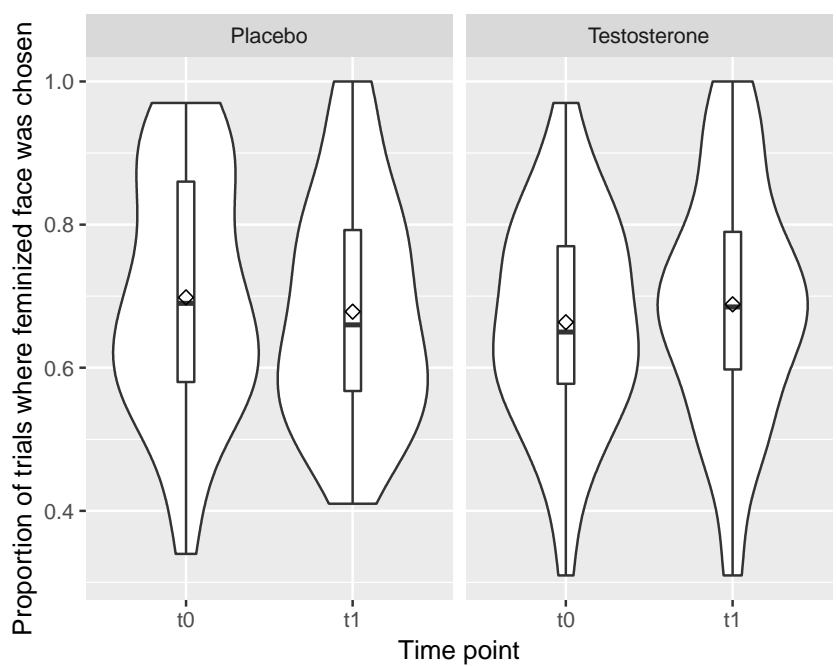

Fig. 1. (A) Example of feminized (left) and masculinized (right) face images. (B) Proportion of trials where feminized face was chosen as function of treatment (Placebo vs. Testosterone) and time point ( $\mathrm{t} 0$ vs. $\mathrm{t} 1$ ). A significant difference was found between $\mathrm{t} 0$ and $\mathrm{t} 1$ in testosterone administration group, but not in the placebo group.

\subsection{Procedure}

Each participant arrived at the laboratory between 11:00 and 14:00. After completing an informed consent form, they were randomly assigned to one of two experimental conditions 
(Treatment): a single dose of testosterone [Androgel ${ }^{\circledR}$ ] or placebo gel. We used a double-blind, placebo-controlled and between-subjects design (i.e., each participant only came to the lab once).

\subsection{Testosterone administration}

Participants in the testosterone condition received a single dose of testosterone gel, containing $150 \mathrm{mg}$ testosterone [Androgel ${ }^{\circledR}$ ], while those in the placebo condition received a colorless hydroalcoholic gel. In both conditions, a trained male research assistant, who was blind to both the study purpose and the experimental condition, applied the gel to the upper arms and shoulders of participants. During the waiting period, participants rested in the testing cubicles and were provided with books and newspapers.

\subsection{Face preference task}

The face preference task consisted of two tasks, one assessing 50 pairs of women's face for attractiveness in short-term mating context, and the other assessing 50 pairs of women's face for attractiveness in the long-term mating context. The descriptions of short-term and long-term mating contexts were similar to previous research (e.g., Bird et al., 2016; Welling et al., 2013). Each pair of faces consisted of masculinized and feminized versions of the same individual (See Fig. 1A). Participants were asked to choose the face in each pair that was more attractive. The order of the faces was randomized, and the feminized face appeared on each side of the screen equally often. Furthermore, we conducted the mate preference task twice (i.e., within-subject design), once at the beginning of the session ( $\mathrm{t} 0$ ) and once 3 hours after gel administration (t1). These time points were chosen based on previous pharmacokinetic data that men's testosterone levels peaked 3 hours after Androgel administration (Eisenegger et al., 2013; Wu et al., 2018).

\subsection{Statistical analysis}

Response on the attractiveness-judgment task was analyzed using mixed binary logistic regression analyses in R Version 3.6.0 (R Core Team, 2019), with lme4 Version 1.1.21 (Bates, Maechler, Bolker, \& Walker, 2015) and lmerTest version 3.1.0 (Kuznetsova, Brockhoff, \& 
Christensen, 2017). Before the analysis, variables were re-coded. The dependent variable of the forced-choice option was a binary choice $(0=$ masculinized face, $1=$ feminized face $)$. Independent variables were coded as, treatment: placebo (- 0.5) vs. testosterone (+ 0.5); mating context: short-term (- 0.5) vs. long-term (+ 0.5); time point: t0 (- 0.5) vs. t1 (+ 0.5). In the model, treatment, time point, and the interaction between treatment and time point were entered as fixed-effect factors. Additionally, given that Bird et al. (2016) demonstrated mating context interacted with the effect of testosterone on shifting facial femininity preferences, mating context and the interaction among mating context, treatment, and time point were entered into the model as well. Subject ID and face ID were entered into the model as random-effect factors.

\section{Results}

The intercept was significant (beta $=0.95, S E=0.09, z=11.06, p<.001$ ), indicating that, on average, Chinese male participants preferred feminine female faces over masculine female faces. The interaction between treatment and time point was significant $($ beta $=0.29, S E=$ $0.10, z=2.77, p=.006$ ). No other main effects or interactions were significant (all absolute betas $<0.09$, all absolute $S E s>0.05$, all absolute $z s<0.77$, all $p s>.441$, see table 1 for details).

Further analysis on the interaction between treatment and time point revealed that, within the testosterone administration group, the facial femininity preferences were significantly different (beta $=0.16, S E=0.07, \mathrm{z}=2.39, p=.017$, Cohen's $d=0.32$ ), and increased from t0 (ratio of feminized face was chosen $M=0.66, S E=0.02$ ) to t1 (ratio of feminized face was chosen $M=0.69, S E=0.02$ ). Within the placebo group, the facial femininity preferences were not significantly different ( beta $=-0.11, S E=0.08, z=-1.42, p=.157$, Cohen's $d=0.27$ ) between the two time points (t0: $M=0.70, S E=0.02$; t1: $M=0.68, S E=0.02)$ (See Fig. 1B). There were no significant differences between two treatment groups at t0 (beta $=-0.23, S E=$ $0.15, z=-1.58, p=.113)$ or at $\mathrm{t} 1($ beta $=0.07, S E=0.17, z=0.38, p=.702)$. 
Moreover, we run additional analyses to control for baseline differences in the femininity preferences. Specifically, we first run a regression analysis that regresses $\mathrm{t} 1$ femininity preferences onto t0 femininity preference (model 1) and then included treatment variable on the next step of the regression model (model 2). Results revealed that model 2 significantly improved the fit of the model to the data compared to model $1, F(1,133)=5.08, p=.026$, and the improvement of R square is from $59.86 \%$ to $61.05 \%$.

Table 1. The results of mixed binary logistic regression of the effect of testosterone on facial femininity preferences

\begin{tabular}{cccccc}
\hline & Beta & $95 \% \mathrm{CI}$ & $\mathrm{SE}$ & $\mathrm{z}$ & $p$ \\
\hline Intercept & 0.95 & $0.78,1.11$ & 0.09 & 11.06 & $<.001$ \\
Treatment & -0.09 & $-0.39,0.21$ & 0.15 & -0.57 & .570 \\
Mating context & -0.07 & $-0.23,0.10$ & 0.09 & -0.77 & .441 \\
Time point & 0.03 & $-0.08,0.14$ & 0.05 & 0.53 & .598 \\
Treatment x Time point & 0.29 & $0.09,0.49$ & 0.10 & 2.77 & .006 \\
Treatment x Time point x Mating context & 0.07 & $-0.15,0.30$ & 0.11 & 0.66 & .511 \\
\hline
\end{tabular}

\section{Discussion}

The present study investigated whether testosterone has an impact on men's preferences for women's facial dimorphism. The results indicated that Chinese men preferred femininity over masculinity in women's faces, and this preference was increased in those given testosterone. Preferences did not change in the placebo group, suggesting that exogenous testosterone has an impact on men's facial femininity preferences. These results provide direct evidence that testosterone has a causal effect on men's facial preferences.

Past research was mostly conducted within Western populations, which may not necessarily apply to other populations since some studies have reported cultural differences in facial attractiveness judgments (DeBruine et al., 2010; Han et al., 2018; Marcinkowska et al., 2014; 
Scott et al., 2014). The current study provided further evidence for the common finding that men are attracted to feminine female faces (Marcinkowska et al., 2014) in China.

The observed change in preferences for feminine women faces after testosterone administration is in line with previous work, suggesting that men with high testosterone levels had a stronger attraction to feminine women (Welling et al., 2008, 2013), regardless of the mating context. However, we note that our results are inconsistent with those of Bird et al. (2016) that the testosterone administration did not enhance preferences for feminine women faces in short-term relationships and rather reduced femininity preferences in long-term relationships. The inconsistencies might be due to the ceiling effect in Bird et al. (2016), which was mentioned in their discussion part. Compared to Bird et al. (2016), which had high baseline (i.e., the number of feminine faces chosen in placebo group were around $85 \%$ ), the baseline in the current study was lower (testosterone administration group around 66\% at t0) and similar to the results in Welling et al. (2008) (low testosterone session around 65\%). As a result, there was little room for increases in feminity preferences in Bird et al. (2016), whereas an increase is more likely to occur when the baseline is relatively low. Similarly, in the current study, a decrease of femininity preferences in the placebo group from t0 to 1 (although not significantly) could be due to the fact that the baseline of femininity preferences in the placebo group was relatively high.

Indeed, the baseline of femininity preferences in the placebo group (70\%) was slightly higher than in the testosterone administration group (66\%), although it was not statistically significant. It is plausible that the relatively higher level of baseline in the placebo group could obscure the effect of testosterone on femininity preference between the two groups (i.e., there were no significant femininity preference differences between the two groups at $\mathrm{t} 1$ ). This may explain why we only observed the effect of testosterone within group/subjects but not between groups. Therefore, future studies should be particularly cautious about baseline differences when interpreting any between-group effect. 
One explanation for our findings could be that testosterone influences femininity preferences via men's risk taking behavior. Given that feminine women are healthier and perceived as more attractive (Thornhill \& Gangestad, 2006), intra-sexual competition among men will be more intense when competing for highly feminine women. Risk-taking behaviors are positively associated with individuals' testosterone levels (Kurath \& Mata, 2018). Compared with low testosterone men, high testosterone men may be more willing to take risks to engage in intra-sexual competition for highly feminine women. Therefore, the stronger preference for feminine women after testosterone administration could be due to the increased risk-taking tendency. As no measures of risk-taking behavior tendency were taken, this explanation remains speculative. Future research could fruitfully investigate the psychological mechanism by which men's preference for feminine women is influenced by testosterone administration.

\section{Role of funding source}

This work was supported by the National Natural Science Foundation of China (31872784, 31600923, 31600928), Guangdong Educational Science Research Fund (2018GXJK150), and the Shenzhen Peacock Plan (827-000233) to YW. The funding sources had no further role in the study design, data collection, analysis, interpretation, and decision to submit this manuscript for publication.

\section{Author contributions}

$\mathrm{CH}$ and $\mathrm{YW}$ designed the study. $\mathrm{ZY}, \mathrm{CH}$, and $\mathrm{YW}$ collected the data. $\mathrm{CH}, \mathrm{YW}$, and $\mathrm{XL}$ analyzed the data. $\mathrm{CH}$ wrote the first version of the paper, XL and EM provided critical revisions. All authors approved the final version for submission.

\section{Acknowledgement}


The authors are grateful to Mr. Jiajun Liao, Mr. Jianxin Ou, Ms. Yu Nan, and Ms. Ran Wei for their help with the study. 


\section{Reference}

Bates, D., Mächler, M., Bolker, B., \& Walker, S. (2015). Fitting Linear Mixed-Effects Models Using lme4. Journal of Statistical Software, 67(1), 1-48.

Bird, B. M., Welling, L. L., Ortiz, T. L., Moreau, B. J., Hansen, S., Emond, M., ... \& Carré, J. M. (2016). Effects of exogenous testosterone and mating context on men's preferences for female facial femininity. Hormones and Behavior, 85, 76-85.

DeBruine, L. M., Jones, B. C., Crawford, J. R., Welling, L. L., \& Little, A. C. (2010). The health of a nation predicts their mate preferences: cross-cultural variation in women's preferences for masculinized male faces. Proceedings of the Royal Society B: Biological Sciences, 277(1692), 2405-2410.

Eisenegger, C., von Eckardstein, A., Fehr, E., \& von Eckardstein, S. (2013).

Pharmacokinetics of testosterone and estradiol gel preparations in healthy young men. Psychoneuroendocrinology, 38(2), 171-178.

Gangestad, S. W., \& Thornhill, R. (2008). Human oestrus. Proceedings of the Royal Society B: Biological Sciences, 275(1638), 991-1000.

Gildersleeve, K., Haselton, M. G., \& Fales, M. R. (2014). Do women's mate preferences change across the ovulatory cycle? A meta-analytic review. Psychological Bulletin, 140(5), 1205.

Han, C., Wang, H., Hahn, A. C., Fisher, C. I., Kandrik, M., Fasolt, V., ... \& Jones, B. C. (2018). Cultural differences in preferences for facial coloration. Evolution and Human Behavior, 39(2), 154-159.

Jones, B. C., Hahn, A. C., Fisher, C. I., Wang, H., Kandrik, M., Han, C., ... \& O’Shea, K. J. (2018). No compelling evidence that preferences for facial masculinity track changes in women's hormonal status. Psychological science, 29(6), 996-1005.

Marcinkowska, U. M., Galbarczyk, A., \& Jasienska, G. (2018). La donna è mobile? Lack of cyclical shifts in facial symmetry, and facial and body masculinity preferences-A hormone based study. Psychoneuroendocrinology, 88, 47-53.

Marcinkowska, U. M., Kozlov, M. V., Cai, H., Contreras-Garduño, J., Dixson, B. J., Oana, G. A., ... \& Prasai, K. (2014). Cross-cultural variation in men's preference for sexual dimorphism in women's faces. Biology letters, 10(4), 20130850. 
Marcinkowska, U. M., Kaminski, G., Little, A. C., \& Jasienska, G. (2018). Average ovarian hormone levels, rather than daily values and their fluctuations, are related to facial preferences among women. Hormones and behavior, 102, 114-119.

Kurath, J., \& Mata, R. (2018). Individual differences in risk taking and endogeneous levels of testosterone, estradiol, and cortisol: A systematic literature search and three independent meta-analyses. Neuroscience \& Biobehavioral Reviews, 90, 428-446.

Kuznetsova, A., Brockhoff, P. B., \& Christensen, R. H. B. (2017). lmerTest package: tests in linear mixed effects models. Journal of Statistical Software, 82(13).

Little, A. C., Jones, B. C., \& DeBruine, L. M. (2011). Facial attractiveness: evolutionary based research. Philosophical Transactions of the Royal Society B: Biological Sciences, $366(1571), 1638-1659$.

R Core Team (2019). R: A language and environment for statistical computing. R Foundation for Statistical Computing, Vienna, Austria.

Scott, I. M., Clark, A. P., Josephson, S. C., Boyette, A. H., Cuthill, I. C., Fried, R. L., ... \& Penton-Voak, I.S. (2014). Human preferences for sexually dimorphic faces may be evolutionarily novel. Proceedings of the National Academy of Sciences, 111(40), 14388-14393.

Thornhill, R., \& Gangestad, S. W. (2006). Facial sexual dimorphism, developmental stability, and susceptibility to disease in men and women. Evolution and Human Behavior, 27 (2), $131-144$.

Welling, L. L., Jones, B. C., DeBruine, L. M., Smith, F. G., Feinberg, D. R., Little, A. C., \& Al-Dujaili, E. A. (2008). Men report stronger attraction to femininity in women's faces when their testosterone levels are high. Hormones and Behavior, 54(5), 703-708.

Welling, L. L., Persola, L., Wheatley, J. R., Cárdenas, R. A., \& Puts, D. A. (2013). Competition and men's face preferences. Personality and Individual Differences, 54(3), 414-419.

Wood, W., Kressel, L., Joshi, P. D., \& Louie, B. (2014). Meta-analysis of menstrual cycle effects on women's mate preferences. Emotion Review, 6(3), 229-249.

Wu, Y., Clark, L., Zilioli, S., Eisenegger, C., Gillan, C. M., Deng, H., \& Li, H. (2018). Single dose testosterone administration modulates emotional reactivity and counterfactual 
choice in healthy males. Psychoneuroendocrinology, 90, 127-133. 


\section{Figure caption}

Fig. 1. (A) Example of feminized (left) and masculinized (right) face images. (B) Proportion of trials where feminized face was chosen as function of treatment (Placebo vs. Testosterone) and time point ( $\mathrm{t} 0$ vs. $\mathrm{t} 1$ ). A significant difference was found between $\mathrm{t} 0$ and $\mathrm{t} 1$ in testosterone administration group, but not in the placebo group. 